Shirley Chang*, OMS IV, Jason Maddox, OMS IV, Erich Berg, OMS IV, Karen Kim, OMS III, Scott Messier, OMS IV, Loren Swanson, OMS IV, Richard Dobrusin, DO, Amy B. Stein, PhD, G. Nathan Nakken, DO, Jeannine Noble, PT and Randall Nydam, PhD

\title{
Evaluating for a correlation between osteopathic examination and ultrasonography on thoracic spine asymmetry
}

https://doi.org/10.1515/jom-2021-0020

Received January 14, 2021; accepted August 9, 2021; published online October 13, 2021

\section{Abstract}

Context: The thoracic spine is a common area of focus in osteopathic manipulative medicine (OMM) for a variety of conditions. Thoracic spine somatic dysfunction diagnosis is achieved by palpating for asymmetry at the tips of the transverse processes (TPs). Previous studies reveal that instead of following the rule of threes, the TPs of a given thoracic vertebra generally align with the spinous process (SP) of the vertebra above. Ultrasonography has been widely utilized as a diagnostic tool to monitor musculoskeletal conditions; it does not utilize ionizing radiation, and it has comparable results to gold-standard modalities. In the case of thoracic somatic dysfunction, ultrasound (US) can be utilized to determine the location of each vertebral TP and its relationship with the SP. Previous studies have investigated the correlation between OMM and ultrasonography of the cervical, lumbar, and sacral regions. However, there has been no study yet that has

\footnotetext{
*Corresponding author: Shirley Chang, OMS IV, Department of Osteopathic Manipulative Medicine, Midwesetern University, Arizona College of Osteopathic Medicine, 19555 N 59th Avenue, Glendale, AZ 85308, USA, E-mail: shirleychang92@gmail.com

Jason Maddox, OMS IV, Erich Berg, OMS IV, Karen Kim, OMS III, Scott Messier, OMS IV and Loren Swanson, OMS IV, Midwestern University, Arizona College of Osteopathic Medicine, Glendale, AZ, USA

Richard Dobrusin, DO and G. Nathan Nakken, DO, Department of Osteopathic Manipulative Medicine, Midwesetern University, Arizona College of Osteopathic Medicine, Glendale, AZ, USA

Amy B. Stein, PhD, Office of Research and Sponsored Programs, Midwesetern University, Glendale, AZ, USA

Jeannine Noble, PT and Randall Nydam, PhD, Department of Anatomy, Midwesetern University, Arizona College of Osteopathic Medicine, Glendale, AZ, USA
}

compared osteopathic structural examination with ultrasonographic examination of the thoracic vertebral region. Objectives: To examine the relationship between osteopathic palpation and ultrasonographic measurements of the thoracic spine by creating a study design that utilizes interexaminer agreement and correlation.

Methods: The ClinicalTrials.gov study identifier is NCT04823637. Subjects were student volunteers recruited from the Midwestern University (MWU)-Glendale campus. A nontoxic, nonpermanent marker was utilized to mark bony landmarks on the skin. Two neuromusculoskeletal boardcertified physicians (OMM1, OMM2) separately performed structural exams by palpating T2-T5 TPs to determine vertebral rotation. Two sonographers (US1, US2) separately scanned and measured the distance from the tip of the SP to the adjacent TPs of the vertebral segment below. Demographic variables were summarized with mean and standard deviation. Interexaminer agreement was assessed with percent agreement, Cohen's Kappa, and Fleiss' Kappa. Correlation was measured by Spearman's rank correlation coefficient. Recruitment and protocols were approved by the MWU Institutional Review Board (IRB).

Results: US had fair interexaminer agreement for the overall most prominent segmental rotation of the T3-T5 thoracic spine, with Cohen's Kappa at $0.27(0.09,0.45)$, and a total agreement percentage at $51.5 \%$. Osteopathic palpation revealed low interexaminer agreement for the overall most prominent vertebral rotation, with Cohen's Kappa at $0.05(0.0,0.27)$, and 31.8\%. Segment-specific vertebral analysis revealed slight agreement between US examiners, with a correlation coefficient of 0.23 , whereas all other pairwise comparisons showed low agreement and correlation. At T4, US had slight interexaminer agreement with 0.24 correlation coefficient, and osteopathic palpation showed low interexaminer (OMM1 vs. OMM2) agreement (0.17 correlation coefficient). At T5, there was moderate agreement between the two sonographers with $0.44(0.27,0.60)$ and 
$63.6 \%$, with a correlation coefficient of 0.57 , and slight agreement between OMM1 and OMM2 with $0.12(0.0,0.28)$ and $42.4 \%$, with 0.23 correlation coefficient.

Conclusions: This preliminary study of an asymptomatic population revealed that there is a low-to-moderate interexaminer reliability between sonographers, low-to-slight interexaminer reliability between osteopathic physicians, and low interexaminer reliability between OMM palpatory examination and ultrasonographic evaluation of the thoracic spine.

Keywords: interexaminer reliability; osteopathic structural exam; spinous process; thoracic spine; transverse process; ultrasound.

Thoracic spinal pain, although less common than lumbar and cervical spinal pain, can be disabling, with a prevalence of $13-15 \%$ in the general population $[1,2]$. Consequently, the thoracic spine is a common area of focus in osteopathic medical schools, and it is an area of treatment focus utilizing osteopathic manipulative medicine (OMM) for a variety of conditions, such as spinal or rib dysfunctions, autonomic nervous system imbalances, and dermatological disease [1, 3-7]. The diagnosis of somatic dysfunction in OMM utilizes the common mnemonic, TART, which stands for tissue texture abnormality, asymmetry in bony positions, restricted range of motion, and tenderness to palpation [8]. Thoracic spine somatic dysfunction diagnosis is achieved by palpating for asymmetry at the tips of the transverse processes (TPs) [7]. The asymmetry in bony positions can be palpated through identification of relationships among anatomical structures, and it is particularly applicable to complaints associated with rib or vertebral displacement [9]. Vertebral asymmetry is indicated by the side with a more posteriorly located TP, which also demonstrates the direction of the vertebral rotation [7]. Accurate diagnosis and naming of somatic dysfunction can be challenging for the early osteopathic student, and interexaminer reliability has often been shown to be low even among advanced practitioners [10-12]. To complicate matters further, the naming of each thoracic vertebral level follows the rule of threes from the model developed by Mitchell et al. [13, 14] However, anatomical studies done by Oakley et al. [13] and Geelhoed et al. [15] as well as radiographic studies reveal that, instead of following the rule of threes, the TPs of a given thoracic vertebra generally align with the spinous process (SP) of the vertebra above [16]. Although there has been an in vivo study conducted that reveals the gross motion of the thoracic spine, to our knowledge, no in vivo study has discussed the anatomical position alignment of the TP with the SP in the thoracic spine [17].
Ultrasonography can be utilized to visualize and to objectively measure the relationship between neighboring anatomical structures [18]. Musculoskeletal ultrasonography has been widely utilized as a diagnostic tool to monitor conditions of soft tissue, joints, ligaments, and cartilage [19]. Ultrasonography has the advantage of absence of ionizing radiation and has shown comparable results to goldstandard modalities like MRI in some areas of the spine $[1,20,21]$. Although ultrasonography can have limitations in that the examiner must have sufficient anatomical knowledge and proper training prior to scanning, ultrasonography has been shown as a reliable modality to identify SPs and TPs of the spine [20, 22]. In the case of thoracic somatic dysfunction, ultrasound (US) can penetrate through multiple layers of soft tissues to visualize the bony features as distinct hyperechoic signals, which can be utilized to determine the location of each vertebral TP and its relationship with neighboring bone features such as the SP [23].

Previous studies have investigated the relationship between OMM and ultrasonography of the cervical, lumbar, and sacral regions [11, 18, 23-26]. However, to our knowledge, no study has compared osteopathic structural examination with ultrasonographic examination of the thoracic vertebral region. In this preliminary study, the authors seek to explore and compare osteopathic palpatory findings of the thoracic TPs with US measurements when evaluating for thoracic vertebral asymmetry. We hope to create a study design utilizing interexaminer agreement and correlation to examine the relationship between osteopathic palpation and ultrasonographic measurements of the thoracic spine.

\section{Methods}

This study was registered with ClinicalTrials.gov and the study identifier is NCT04823637. The study protocol was approved by the Midwestern University (MWU) Institutional Review Board (IRB) at the Glendale, Arizona campus, and the participants provided written informed consent. This study did not require any funding. The study actively recruited subjects between October 10, 2019 and January 30, 2020. Email announcements were sent to students at the Arizona College of Osteopathic Medicine and the Master's in Biomedical Sciences program at MWU in Glendale, Arizona.

\section{Subject recruitment}

The subjects are MWU students who were recruited via email announcements, with responses being recorded via a 
secure Google Form that was accessible only to the MWU network. Recruitment email announcements were sent out to first- and second-year osteopathic medical students and Master's program students at MWU in Glendale, Arizona. The study was approved by the MWU IRB, and approximately 600 emails were sent out to recruit a maximum of 100 subjects. Participants voluntarily provided information on demographics without attention to race or ethnicity, history of back pain, musculoskeletal structural diseases, and other metrics for exclusionary purposes. A total of 86 students responded affirmatively, and 74 out of those 86 students showed up to participate and continued through data collection after signing informed consents. Individuals were then excluded if they demonstrated a history or current diagnosis of scoliosis, spondylosis, spondylolisthesis, herniated disc, spinal fracture, or surgery to the spine. After the exclusionary criteria were applied, data from 66 subjects were utilized for data analysis.

\section{Setup and landmark marking}

Subjects were seated upright in stable chairs with substantial lumbar support, with the back of the chairs below the level of T7 SP. Each subject was placed in an individual room next to an ultrasound machine and could not communicate with each other while the study was in session. Each examiner was blinded and could not communicate with each other or see what the other examiner was performing throughout the entirety of the data collection phase. There was a data collector stationed at each subject area to record all data, which were blinded from the examiners.

Marking of landmarks on the skin were performed by an OMM Scholar. Members of the OMM Scholarship program are osteopathic students selected through a competitive evaluation system to become peer instructors within the Department of Osteopathic Family and Community Medicine. The OMM Scholar in this study was a fourth-year medical student who was in her second year of teaching structural palpation, osteopathic diagnosis, and treatment techniques to first- and second-year medical students. The OMM Scholar palpated subjects' upper backs on the skin and gave verbal instructions such as "look down" and "look up to the ceiling," to obtain accurate determination of each SP. First, the C7 SP bony protrusion was identified utilizing a nontoxic, non-permanent skin marking pen. Then, the subsequent thoracic SPs of T1-4 were palpated and marked. To mark the TP, the marker is placed at a distance of onehalf inch directly lateral to each SP [7]. Then, each TP is marked with the number of the vertebra below (i.e., next to the T2 SP, the number " 3 " is written to indicate the TP of T3) until the T5 TPs were marked (Figure 1A, B). All vertebral segments indicated in the study, if not otherwise indicated, refer to the TPs of each vertebra (e.g., T3=T3 TP). We have decided to focus on the T3-T5 vertebrae to limit interference from other anatomical structures [27].

\section{OMM structural exam}

The main motion of the thoracic spine is rotation. The range of motion for sidebending, flexion, and extension are limited by the ribcage [28, 29]. Furthermore, small rotatory movements are permitted between adjacent vertebrae due to the position of the SP [30]. Therefore, to ensure reproducibility with the US protocol, and given that the major motion of each thoracic vertebra is rotation, the OMM structural exam focuses on the rotational component of the thoracic spine somatic dysfunction.

Two osteopathic physicians who are neuromusculoskeletal medicine (NMM) certified by the American Osteopathic Board of Nuclear Medicine (AOBNM) performed the osteopathic structural exam portion of the study. Both were OMM faculty members teaching at MWU at the time of the research data collection. Multiple training sessions were conducted prior to the data collection phase. Two examiners conducted the exam to minimize single examiner bias [31]. Prior to each data collection session, the protocol was reviewed by the examiners. Each subject was asked to sit up straight and to look straight ahead at a far point on the wall. The exam was performed at the T2T5 TP region in the neutral spine position initially. Examiners utilized the marked spots as a guide and palpated the marked TPs on the skin. First, the examiner palpated each vertebra from $\mathrm{T} 2$ to $\mathrm{T} 5$ to determine a left or right rotation by palpating for a more posteriorly rotated TP, or a "speedbump" (i.e., the side that had a more noticeable hard or "bony" protrusion feel). Then, the examiner assessed which TPs were rotated based on palpation. To ensure that the protocol is as similar to the ultrasonography protocol as possible and to prevent the possibility that a somatic dysfunction may be "corrected" during the structural exam, motion testing through neck flexion and extension was only introduced to confirm findings when the examiner felt absolutely necessary. Finally, the physician identified the most prominently rotated vertebra that is clinically treatable for each subject. Once the first examiner had completed the exam, a second examiner repeated the above steps on the same subject. 

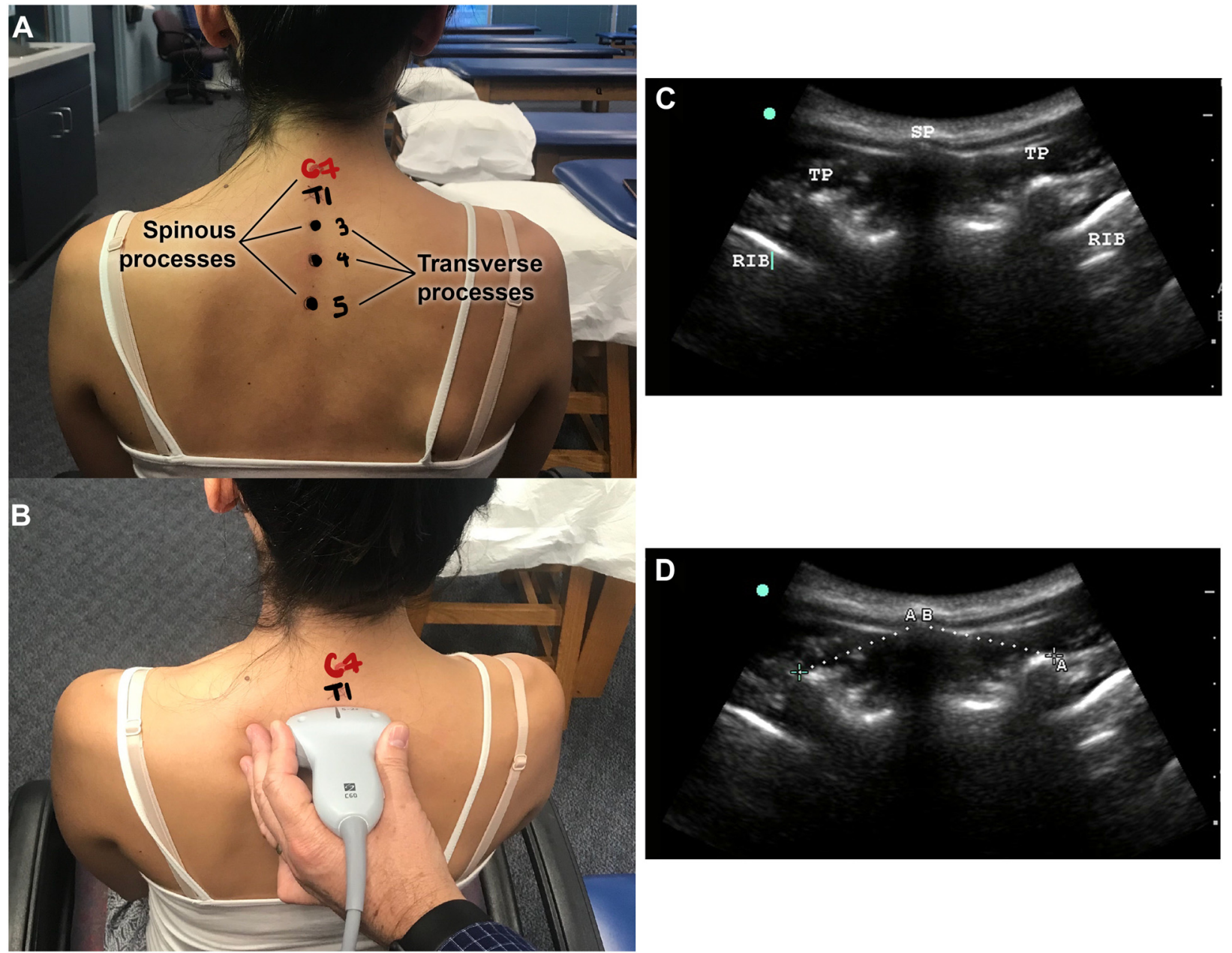

Figure 1: (A) Demonstration picture showing how the subjects are marked. The C7 SP is marked as "C7" in red, the T1 SP is marked as "T1" in black, and the SPs of T2-T4 are marked with black dots. The T3 TP is marked as "3," the T4 TP is marked as "4," and the T5 TP is marked as "5." (B) Demonstration picture showing that the US probe is placed perpendicular to the SP of the vertebra above and the TPs of the vertebra under examination. (C) US imaging with identifications of landmarks. SP, spinous process of the vertebra above; TP, transverse process under examination. The ribs are also identified. (D) US imaging showing how measurements are achieved. The SP is identified, and the distance between the SP of the vertebra above to the TPs is measured. SP, spinous process; TP, transverse process; US, ultrasound.

The OMM portion was complete after both OMM physicians concluded their structural exam. During data collection, the osteopathic physicians were blinded to the examination findings of each other and the findings of the sonographers.

\section{Ultrasound exam}

US exam was performed by two sonographers after the completion of the OMM examination. One sonographer is certified by the ARDMS in musculoskeletal (Registered in Musculoskeletal [RMSK]) ultrasound (US1), and the other sonographer is a long-time anatomy professor (US2) with extensive US training who has taught US to medical students as a part of the anatomy and OMM curriculum since
2017 at MWU. The protocol was developed under supervision of the certified musculoskeletal US specialist. Multiple training sessions were conducted prior to the data collection phase. Two examiners conducted the exam to minimize single examiner bias. Prior to each data collection session, the protocol was reviewed by the sonographers. Scans were performed with curvilinear probes, and the depth was adjusted to maximize the view of the neural arch region of the vertebrae. The subjects were seated upright and relaxed with lumbar support, with marked spots still visible.

\section{Ultrasound scanning}

The scan was performed with curvilinear probes in the transverse view. Sonographers started by placing the probe 
at the T2 SP, which appeared as the most hyperechoic section at the top of the screen. After the T2 SP was visualized, the tip of the SP was confirmed by dropping the probe below T2 SP until it was no longer visualized, and then the probe was moved back up again until the tip of the T2 SP was visualized again. Then, the probe was angled such that the T3 TPs and the T2 SP were all visualized in the same plane. The image was then frozen to measure the distance between T2 SP and each of the T3 TPs utilizing the caliper function of the US machine. This was repeated for T4 TPs and T5 TPs. Once the first sonographer was done, the process was repeated with the second sonographer. During data collection, the sonographers were blinded to the examination and findings of each other and the osteopathic physicians.

\section{Ultrasound measuring}

The integrated caliper function was utilized to determine the measurement from the center of SP to the "tallest" point (i.e., lateral apex) of the TP of the vertebral segment below on the right side of the screen first. The same process was repeated for the left side of the screen. The data were then recorded in centimeters $(\mathrm{cm})$. The lower measurement (smaller number) indicated the direction of the rotation (Figure 1C, D).

\section{Data documentation/variable calculation}

\section{OMM data}

OMM documentation indicated each segment as left (L) or right $(\mathrm{R})$ and determined whether each vertebra was "prominent" or not. If the examiner determined that the rotation is prominent enough to be felt by palpation, the data recorder checked off "yes" next to the indicated segment. If the examiner determined that the rotation was not prominent, then the recorder checked "no" and that vertebral segment was considered to be in "neutral" position with no rotation.

\section{Ultrasound data}

US measurements of both the left (L) and right (R) sides were documented. Vertebral rotation would cause the TP of the involved side to be more posteriorly located and thus closer in distance to the SP of the segment above [32]. This phenomenon was measured on US imaging as the side that has a smaller distance between the involved TP side and the SP of the segment above (Figure 1C, D). To obtain a value that can be utilized for variable calculation, we arbitrarily designated $\mathrm{R}$ as the reference point and calculated the differences of rotation by subtracting the measurement of $L$ from $R(R-L)$. The numbers are recorded in centimeters $(\mathrm{cm})$. A positive number indicated a left rotation (because L was a smaller number), and a negative number indicated a right rotation (because $\mathrm{R}$ was a smaller number). The highest numerical difference between the two TP measurements determined the most prominently rotated vertebra. To obtain the threshold of rotation to determine "neutral," we utilized a normal distribution defining a 95.0\% confidence interval for the mean around 0 . Please see the "Statistical Analysis" section below for more details.

\section{Statistical analysis}

Demographic variables were summarized with the mean and standard deviation (SD). Average US measurement differences were calculated utilizing Microsoft Excel functions. The US measurements were defined as dysfunctional for each vertebra by defining a $95 \%$ confidence interval for the mean around 0. Measurement differences outside of the confidence interval were considered dysfunctional and specified as right or left depending on a positive or negative difference value. Differences within the interval were defined to be neutral. Differences in mean US measurements were analyzed with a paired t-test. Interexaminer reliability for both ultrasonography and OMM modalities were assessed with total agreement percentage, Cohen's Kappa, and Fleiss' Kappa. Utilizing Cohen's Kappa and Fleiss' Kappa in addition to agreement percentages allowed for the correction of examiner agreement due to chance. Cohen's and Fleiss' Kappa were interpreted as follows: 0.10-0.20 indicated slight agreement, $0.21-0.40$ indicated fair agreement, $0.41-0.60$ indicated moderate agreement, 0.61-0.80 indicated substantial agreement, and 0.81-0.99 indicated near perfect agreement. Validity between the US and OMM measurements was assessed utilizing the Spearman's rank correlation coefficient. The Spearman correlation is a nonparametric measure of rank correlation and assesses the strength of a monotonic relationship. Generally, Spearman's rank correlation describes the strength of correlation by utilizing the following absolute values: $0.00-0.19$ as "very low," $0.20-0.39$ as "low," $0.40-0.59$ as "moderate," 0.5-0.79 as "strong," and 0.80-1.0 as "very strong." [33] Statistical analysis was done utilizing R version 3.6.2, and statistical significance was assessed at the $p<0.05$ level. 

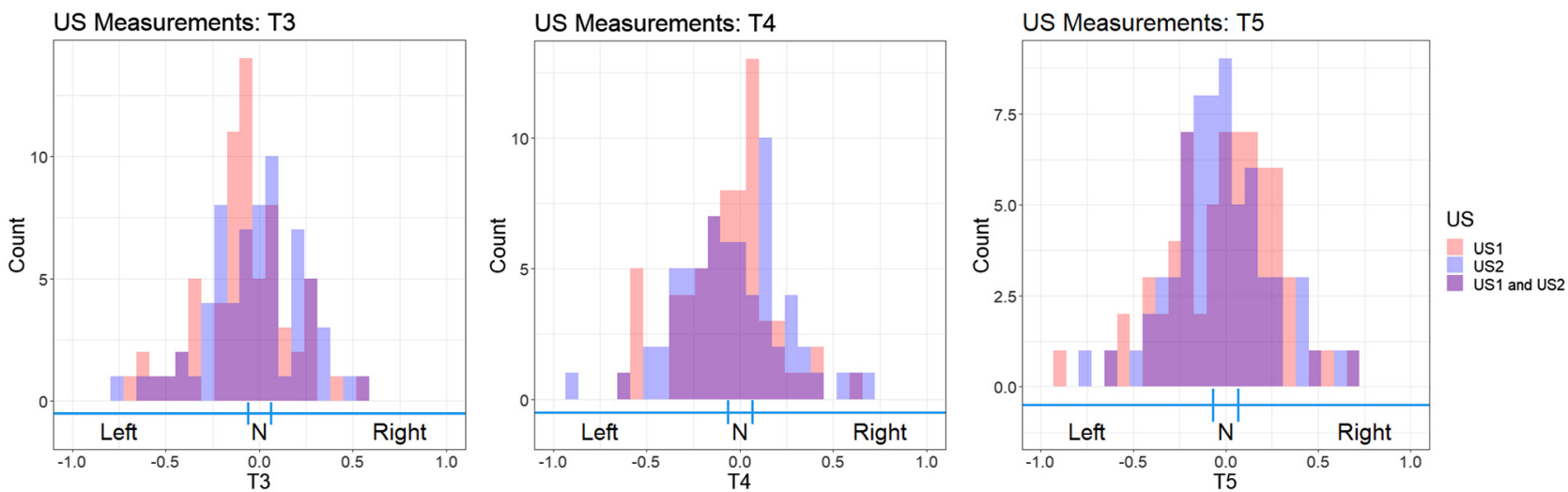

Figure 2: Histogram of the US measurement difference for each vertebral segment, T3, T4, and T5. The blue line underneath represents the $95 \%$ confidence interval for each segment. Within the interval was classified as neutral (N), below as left, and above as right. US, ultrasound.

\section{Consideration of the rotated superior vertebra in a 2-vertebrae segment in the comparison of ultrasonography and $\mathrm{OMM}$ findings}

One consideration in the comparison between ultrasonography and OMM is that the superior vertebra, if rotated, may displace the position of the SP from the midline position, leading to changes in the distance from that SP to the TP of the vertebra immediately below. To ensure that this phenomenon is also taken into consideration when analyzing the reliability between ultrasonography and OMM, we also analyzed the vertebral rotations by utilizing SP as the reference point. A rotated vertebra changes the position of the TPs and SP such that the SP would be located contralateral to the direction of the vertebral rotation and the TP that is more posteriorly located [34]. Because OMM palpated the TPs from T2-T5, we can take the position of the TPs to infer the SP position, which would be contralateral to the TP position of the same vertebra (i.e., if the examiner finds a posterior right $\mathrm{T} 3 \mathrm{TP}$, then the T3 SP would be displaced to the left, and therefore labeled as "left"). Ultrasonography utilized the SP of the superior vertebra and the TPs of the inferior vertebra as the endpoints for the distance measurement. Thus, in this alternative analysis, the ultrasonography measurements of T3T5 TPs are relabeled to match the SP of the vertebra above (i.e., T2 SP, T3 SP, and T4 SP). Interexaminer reliability between ultrasonography and OMM of each SP of the T2T4 vertebrae was then assessed with the total agreement percentage, Cohen's Kappa, and Fleiss' Kappa.

\section{Results}

The overall demographics of participants showed 31 (47.0\%) males and 35 (53.0\%) females. Race and ethnicity were not collected for the purpose of this study because they were not variables being evaluated or investigated. The subjects' age range is 22-35 years of age, with the mean age as 26.61 years (SD, 3.1) for males and 25.71 years (SD, 2.7) for females. The BMI range is $15.9-36.9 \mathrm{~kg} / \mathrm{m}^{2}$, with the mean BMI as $24.13 \mathrm{~kg} / \mathrm{m}^{2}$ (SD, 4.2) overall, $25.46 \mathrm{~kg} / \mathrm{m}^{2}$ (SD, 3.5) for males, and $22.95 \mathrm{~kg} / \mathrm{m}^{2}$ (SD, 4.5) for females.

The average US measurement differences for both US1 and US2 is $0.207 \mathrm{~cm}$ or $2.07 \mathrm{~mm}$ (see Supplemental section for raw data). The mean US measurement between US1 and US2 across all participants was found to have no significant difference at T3 $(p=0.1523), \mathrm{T} 4 \quad(\mathrm{p}=0.5029)$, and $\mathrm{T} 5$ $(p=0.4712)$. Ultrasonography measurements for $\mathrm{T} 3, \mathrm{~T} 4$, and T5 were normally distributed around a 95\% confidence interval, with a margin of error of 0.062 at T3, 0.066 at T4, and 0.068 at T5 (Figure 2).

Our results showed that ultrasonography had fair interexaminer agreement between US1 and US2 for the overall "most prominent" segmental rotation of the thoracic spine, with Cohen's Kappa at 0.27 , with range of $(0.09,0.45)$, and total agreement percentage at a 51.5\% (Figure 3A, B). For osteopathic examination (OMM), there is low statistical interexaminer agreement between OMM1 and OMM2 for the overall thoracic spine, with a Cohen's Kappa at $0.05(0.0,0.27)$ and $31.8 \%$. Both US1 and US2 had slight agreement with one of the osteopathic examiners (OMM2), with Cohen's Kappa of $0.14(0.0,0.33), 31.8 \%$ for 


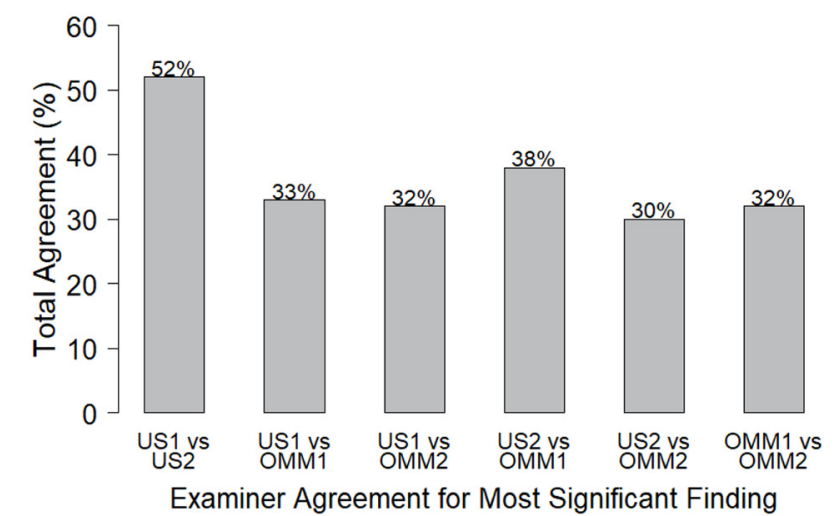

A

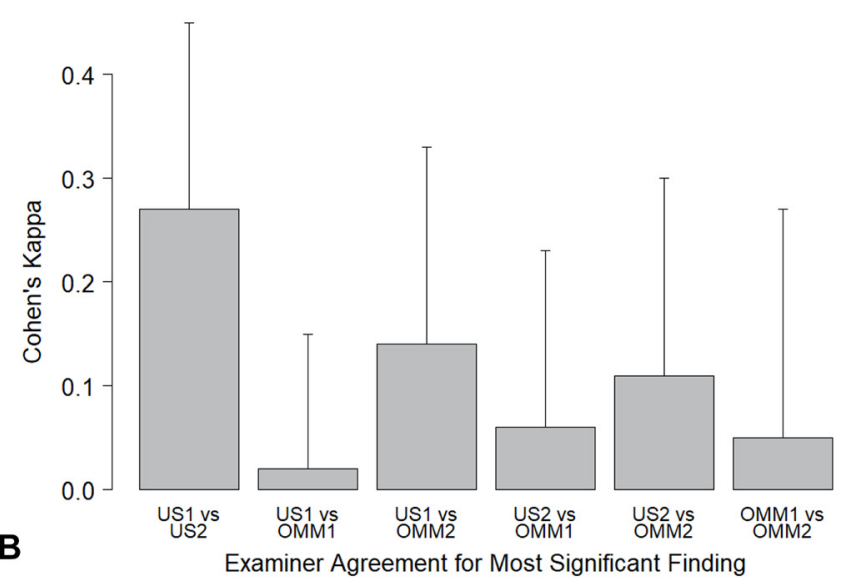

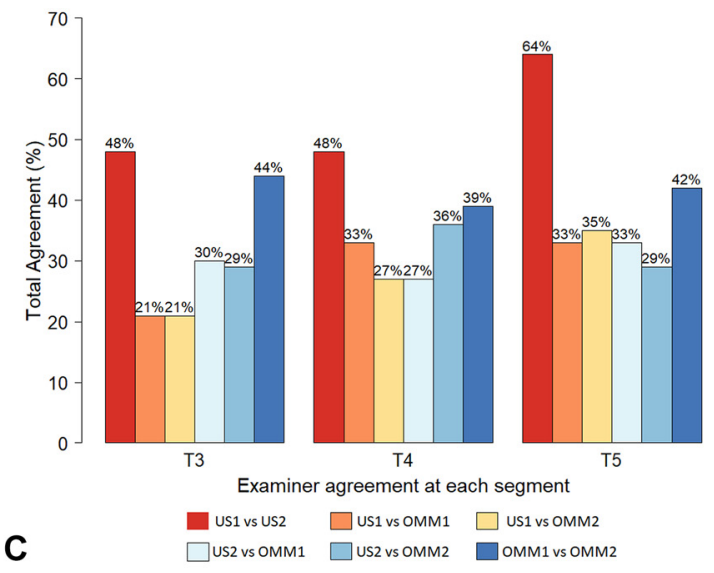

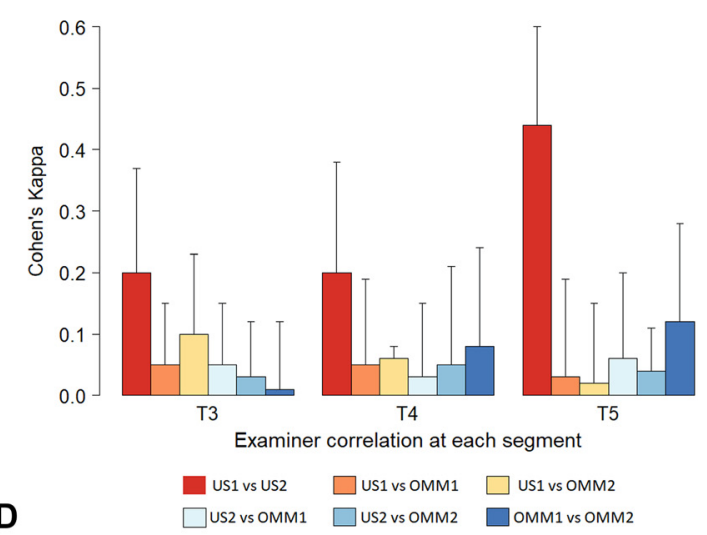

Figure 3: (A) Bar plot of the total agreement comparing the most prominent (significant) findings as indicated by US measurement and OMM palpatory findings. (B) Bar plot of Cohen's Kappa values for each pairwise comparison for the most significant/overall findings. Cohen's Kappa is utilized to examine interexaminer agreement. The error bar is representative of the $95 \%$ confidence interval for Cohen's Kappa. We interpret Cohen's Kappa as $0.10-0.20$ is indicative of slight agreement, $0.21-0.40$ of fair agreement; $0.41-0.60$ of moderate agreement; $0.61-0.80$ of substantial agreement; and 0.81-0.99 of near-perfect agreement. (C) Bar plot of the total agreement between OMM and US examiners, for each pairwise comparison. (D) Bar plot of Cohen's Kappa between OMM and US examiners, for each pairwise comparison at T3, T4, and T5. The error bar is representative of the $95 \%$ confidence interval for Cohen's Kappa. We interpret Cohen's Kappa as $0.10-0.20$ is indicative of slight agreement, $0.21-0.40$ of fair agreement; $0.41-0.60$ of moderate agreement; $0.61-0.80$ of substantial agreement; and $0.81-0.99$ of nearperfect agreement. OMM, osteopathic manipulative medicine; US, ultrasound.

US1 vs. OMM2 and $0.11(0.0,0.30), 30.3 \%$ for US2 vs. OMM2 (Table 1A).

However, individual segmental analysis reveals that at T3, US1 and US2 has slight agreement at $0.20(0.02,0.37)$ and $48.5 \%$ calculated via Cohen's Kappa and total agreement percentage, with a correlation coefficient of 0.23 $(-0.02,0.45)$ calculated by Spearman's Rank Correlation. OMM1 and OMM2 showed low agreement at $0.01(0.0,0.12)$ and $43.9 \%$, and a correlation of $-0.04(-0.28,0.20)$ correlation coefficient (Figure 3C, D; Tables 1B and 1D). There is slight agreement between US1 and OMM2 (0.10 [0.03, 0.23]) and 21.2\%. US1 and OMM1, US1 and OMM2, US2 and OMM1, and US2 and OMM2 all showed low levels of agreement, with Cohen's Kappa and total agreement percentage as the following: $0.05(0.0,0.15)$ and $21.2 \%, 0.10(0.03,0.23)$ and $21.2 \%, 0.05(0.0,0.15)$ and $30.3 \%$, and $0.03(0.0,0.12)$ and $28.8 \%$, respectively. US2 and OMM1 had a Spearman correlation of $0.17(0.08,0.40)$, while US1 and OMM1, US1 and OMM2, OMM1 and OMM2, and US2 and OMM2 all had negative correlation values (Table 1D).

At T4, US has slight interexaminer (US1 vs. US2) agreement at $0.2(0.02,0.38)$ and $48.5 \%$, with $0.24(0.0$, 0.46) correlation coefficient, and osteopathic examine showed low interexaminer (OMM1 vs. OMM2) agreement with $0.08(0.0,0.24)$ and $39.4 \%$ with $0.17(-0.08,0.40)$ correlation coefficient. All other pairwise comparisons revealed low levels of agreement: US1 vs. OMM1 showed $0.05(0.0,0.19)$ and $33.3 \%$, US1 vs. OMM2 showed 0.06 
Table 1A: Comparison of US and OMM “most prominent" findings of TPs.

\begin{tabular}{llrr}
\hline $\begin{array}{l}\text { Comparison } \\
\mathbf{1}\end{array}$ & $\begin{array}{l}\text { Comparison } \\
\mathbf{2}\end{array}$ & $\begin{array}{r}\text { Total agreement } \\
\text { \% (actual number) }\end{array}$ & $\begin{array}{r}\text { Cohen's Kappa } \\
\mathbf{( 9 5 \% ~ C l )}\end{array}$ \\
\hline US1 & US2 & $51.5 \%(34 / 66)$ & $0.27(0.09,0.45)$ \\
US1 & OMM1 & $33.3 \%(22 / 66)$ & $0.02(0.0,0.15)$ \\
US1 & OMM2 & $31.8 \%(21 / 66)$ & $0.14(0.0,0.33)$ \\
US2 & OMM1 & $37.9 \%(25 / 66)$ & $0.06(0.0,0.23)$ \\
US2 & OMM2 & $30.3 \%(20 / 66)$ & $0.11(0.0,0.30)$ \\
OMM1 & OMM2 & $31.8 \%(21 / 66)$ & $0.05(0.0,0.27)$ \\
\hline
\end{tabular}

The final analysis looks at comparing the US and the OMM "most significant" findings (red). We again present total agreement, Cohen's Kappa between the pairwise comparisons of most significant findings, and Fleiss' Kappa to compare all four raters at once. Cohen's Kappa interpretation: $0.10-0.20=$ slight agreement; $0.21-0.40=$ fair agreement; $0.41-0.60=$ moderate agreement; $0.61-0.80=$ substantial agreement; and 0.81-0.99=near-perfect agreement. $\mathrm{Cl}$, confidence interval; OMM, osteopathic manipulative medicine; TP, transverse process; US, ultrasound.

$(0.0,0.08)$ and $27.3 \%$, US2 vs. OMM1 showed 0.03 (0.0, $0.15)$ and $27.3 \%$, and US2 vs. OMM2 showed $0.05(0.0,0.21)$ and $36.4 \%$. Those pairwise comparisons (US1 vs. OMM1, US1 vs. OMM2, US2 vs. OMM1, US2 vs. OMM2) also showed low levels of correlation (Figure 3C, D; Tables 1B and 1D).

At T5, ultrasonography had moderate interexaminer agreement at $0.44(0.27,0.60)$, or $63.6 \%$ and moderate correlation of 0.57 (0.36-0.72). OMM had slight interexaminer agreement at $0.12(0.0,0.28)$ and $42.4 \%$, with a correlation coefficient of $0.23(-0.02,0.45)$. All other pairwise comparisons revealed low levels of agreement-US1 vs. OMM1 showed $0.03(0.0,0.19)$ and 33.3\%, US1 vs. OMM2 showed $0.02(0.0,0.15)$ and $34.8 \%$, US2 vs. OMM1 showed $0.06(0.0,0.12)$ and $33.3 \%$, and US2 vs. OMM2 showed 0.04 $(0.0,0.11)$ and $28.8 \%$. Those pairwise comparisons (US1 vs. OMM1, US1 vs. OMM2, US2 vs. OMM1, US2 vs. OMM2) also showed low levels of correlation (Figure 3C, D; Tables 1B and 1D).

Fleiss' Kappa compares all raters at once: US1, US2, OMM1, and OMM2 (66 subjects and 4 raters). Interpretation is similar to Cohen's Kappa. Fleiss' Kappa is -0.0512 at T3, 0.0287 at T4, and 0.0794 at T5, all of which indicate low levels of agreements (Table 1C).

Statistical analysis of ultrasonography and OMM agreement on the SP position revealed low levels of agreement. We found that US1 vs. OMM1 showed a Cohen's Kappa of $0(0,0)$ and total agreement percentage of 21.2\% for T2 SP (Figure 4; Table 1B). For T3 SP, we found 0.03 $(0,0.13)$ and $30.3 \%$, and $0.05(0,0.18)$ and $33.3 \%$ for T4 SP. US1 vs. OMM2 showed agreement of $0.03(0,0.19)$ and $37.9 \%$ for T2 SP, $0.08(0,0.24)$ and $37.9 \%$ for T3 SP, and
Table 1B: Pairwise comparisons including between US and OMM findings for transverse processes and spinous processes positions (left, right, or neutral).

\begin{tabular}{|c|c|c|c|}
\hline Comparison & $\begin{array}{l}\text { Vertebrae } \\
\text { (TP) }\end{array}$ & $\begin{array}{r}\text { Total agreement } \\
\% \text { (actual number) }\end{array}$ & $\begin{array}{r}\text { Cohen's Kappa } \\
(95 \% \mathrm{Cl})\end{array}$ \\
\hline OMM1 vs. & T3 & $43.9 \%(29 / 66)$ & $0.01(0.0,0.12)$ \\
\hline \multirow[t]{2}{*}{ OMM2 } & T4 & $39.4 \%(26 / 66)$ & $0.08(0.0,0.24)$ \\
\hline & T5 & $42.4 \%(28 / 66)$ & $0.12(0.0,0.28)$ \\
\hline \multirow[t]{3}{*}{ US1 vs. US2 } & T3 & $48.5 \%(32 / 66)$ & $\begin{array}{r}0.20(0.02 \\
0.37)\end{array}$ \\
\hline & T4 & $48.5 \%(32 / 66)$ & $\begin{array}{r}0.20(0.02 \\
0.38)\end{array}$ \\
\hline & T5 & $63.6 \%(42 / 66)$ & $\begin{array}{r}0.44(0.27 \\
0.60)\end{array}$ \\
\hline \multirow[t]{3}{*}{ US1 vs. OMM1 } & T3 & $21.2 \%(14 / 66)$ & $0.05(0.0,0.15)$ \\
\hline & $\mathrm{T} 4$ & $33.3 \%(22 / 66)$ & $0.05(0.0,0.19)$ \\
\hline & T5 & $33.3 \%(22 / 66)$ & $0.03(0.0,0.19)$ \\
\hline \multirow[t]{3}{*}{ US1 vs. OMM2 } & T3 & $21.2 \%(14 / 66)$ & $\begin{array}{r}0.10(0.03 \\
0.23)\end{array}$ \\
\hline & T4 & $27.3 \%(18 / 66)$ & $0.06(0.0,0.08)$ \\
\hline & T5 & $34.8 \%(23 / 66)$ & $0.02(0.0,0.15)$ \\
\hline \multirow[t]{3}{*}{ US2 vs. OMM1 } & T3 & $30.3 \%(20 / 66)$ & $0.05(0.0,0.15)$ \\
\hline & T4 & $27.3 \%(18 / 66)$ & $0.03(0.0,0.15)$ \\
\hline & T5 & $33.3 \%(22 / 66)$ & $0.06(0.0,0.20)$ \\
\hline \multirow[t]{3}{*}{ US2 vs. OMM2 } & Т3 & $28.8 \%(19 / 66)$ & $0.03(0.0,0.12)$ \\
\hline & T4 & $36.4 \%(24 / 66)$ & $0.05(0.0,0.21)$ \\
\hline & T5 & $28.8 \%(19 / 66)$ & $0.04(0.0,0.11)$ \\
\hline
\end{tabular}

Comparison of US and OMM findings of T2-4 spinous processes

\begin{tabular}{llrr}
\hline Comparison & $\begin{array}{l}\text { Vertebrae } \\
\text { (SP) }\end{array}$ & Total agreement & $\begin{array}{r}\text { Cohen's Kappa } \\
(95 \% \mathrm{Cl})\end{array}$ \\
\hline US1 vs. OMM1 & T2 & $21.2 \%(14 / 66)$ & $0(0,0)$ \\
& T3 & $30.3 \%(20 / 66)$ & $0.03(0,0.13)$ \\
& T4 & $33.3 \%(22 / 66)$ & $0.05(0,0.18)$ \\
US1 vs. OMM2 & T2 & $37.9 \%(25 / 66)$ & $0.03(0,0.19)$ \\
& T3 & $37.9 \%(25 / 66)$ & $0.08(0,0.24)$ \\
& T4 & $34.8 \%(23 / 66)$ & $0.03(0,0.10)$ \\
US2 vs. OMM1 & T2 & $24.2 \%(16 / 66)$ & $0(0,0)$ \\
& T3 & $18.2 \%(12 / 66)$ & $0.009(0,0.06)$ \\
& T4 & $28.8 \%(19 / 66)$ & $0.06(0,0.17)$ \\
US2 vs. OMM2 & T2 & $27.3 \%(18 / 66)$ & $0.02(0,0.19)$ \\
& T3 & $28.8 \%(19 / 66)$ & $0.02(0,0.13)$ \\
& T4 & $22.7 \%(15 / 66)$ & $0(0,0.05)$ \\
\hline
\end{tabular}

Pairwise comparisons include US1 vs. OMM1, US1 vs. OMM2, US2 vs. OMM1, US2 vs. OMM2, and OMM1 vs. OMM2. Cohen's Kappa and total agreement are reported. $\mathrm{Cl}$, confidence interval; OMM, osteopathic manipulative medicine; SP, spinous process; TP, transverse process; US, ultrasound.

$0.03(0,0.10)$ and $34.8 \%$ for T4 SP. US2 vs. OMM1 showed agreement of $0(0,0)$ and $24.2 \%$ for T2 SP, $0.009(0,0.06)$ and $18.2 \%$ for T3 SP, and $0.06(0,0.17)$ and $28.8 \%$ for T $4 \mathrm{SP}$. US2 vs. OMM2 showed $0.02(0,0.19)$ and $27.3 \%$ for T2 SP, $0.02(0,0.13)$ and $28.8 \%$ for T3 SP, and $0(0,0.05)$ and $22.7 \%$ 
Table 1C. Fleiss' Kappa comparisons of all raters at once: US1, US2, OMM1, and OMM2 (66 subjects and 4 raters).

\begin{tabular}{lrrrr}
\hline $\begin{array}{l}\text { Transverse processes: } \\
\text { US overall vs. OMM } \\
\text { overall using Fleiss" }\end{array}$ & $\begin{array}{r}\text { US vs. OMM overall } \\
\text { “most prominent" } \\
\text { Kappa }\end{array}$ & $\begin{array}{r}\text { Spinous process: US } \\
\text { finding } \\
\text { overall vs. OMM over- } \\
\text { all using Fleiss' Kappa }\end{array}$ \\
\hline Vertebrae & $\begin{array}{r}\text { Fleiss } \\
\text { Kappa }\end{array}$ & & Vertebrae & $\begin{array}{r}\text { Fleiss } \\
\text { Kappa }\end{array}$ \\
\cline { 4 - 5 } & $(95 \% \mathrm{Cl})$ & & & $(95 \% \mathrm{Cl})$ \\
\hline T3 TP & -0.0512 & 0.0689 & T2 SP & -0.0623 \\
T4 TP & 0.0287 & T3 SP & -0.0119 \\
T5 TP & 0.0794 & T4 SP & 0.0338 \\
\hline
\end{tabular}

This table includes all rater comparisons for all the transverse processes, the "most prominent" finding, and the spinous process. Interpretation is similar to Cohen's Kappa. Cl, confidence interval; OMM, osteopathic manipulative medicine; US, ultrasound.

Table 1D. Spearman's rank correlation-TP T3-T5.

\begin{tabular}{|c|c|c|c|}
\hline \multirow[t]{2}{*}{$\begin{array}{l}\text { Transverse } \\
\text { process }\end{array}$} & \multirow[t]{2}{*}{ Comparison } & \multicolumn{2}{|c|}{$\begin{array}{c}\text { Spearman correlation } \\
\text { coefficient }\end{array}$} \\
\hline & & Correlation $(95 \% \mathrm{Cl})$ & p-Value \\
\hline \multirow[t]{6}{*}{ T3 } & US1 vs. US2 & $0.23(-0.02,0.45)$ & 0.0669 \\
\hline & $\begin{array}{l}\text { OMM1 vs. } \\
\text { OMM2 }\end{array}$ & $-0.04(-0.28,0.20)$ & 0.7455 \\
\hline & US1 vs. OMM1 & $-0.13(-0.36,0.12)$ & 0.2931 \\
\hline & US1 vs. OMM2 & $-0.10(-0.33,0.15)$ & 0.4385 \\
\hline & US2 vs. OMM1 & $0.17(-0.08,0.40)$ & 0.1734 \\
\hline & US2 vs. OMM2 & $-0.16(-0.39,0.09)$ & 0.1987 \\
\hline \multirow[t]{6}{*}{ T4 } & US1 vs. US2 & $0.24(0.0,0.46)$ & 0.0475 \\
\hline & $\begin{array}{l}\text { OMM1 vs. } \\
\text { OMM2 }\end{array}$ & $0.17(-0.08,0.40)$ & 0.1771 \\
\hline & US1 vs. OMM1 & $0.02(-0.22,0.27)$ & 0.8431 \\
\hline & US1 vs. OMM2 & $0.04(-0.21,0.27)$ & 0.7797 \\
\hline & US2 vs. OMM1 & $-0.02(-0.26,0.22)$ & 0.8642 \\
\hline & US2 vs. OMM2 & $0.02(-0.22,0.26)$ & 0.8596 \\
\hline \multirow[t]{6}{*}{ T5 } & US1 vs. US2 & $0.57(0.36,0.72)$ & $<0.0001$ \\
\hline & $\begin{array}{l}\text { OMM1 vs. } \\
\text { OMM2 }\end{array}$ & $0.23(-0.02,0.45)$ & 0.0693 \\
\hline & US1 vs. OMM1 & $-0.03(-0.27,0.22)$ & 0.8307 \\
\hline & US1 vs. OMM2 & $0.01(-0.24,0.25)$ & 0.9589 \\
\hline & US2 vs. OMM1 & $0.12(-0.12,0.36)$ & 0.3254 \\
\hline & US2 vs. OMM2 & $-0.02(-0.27,0.22)$ & 0.8444 \\
\hline
\end{tabular}

Measurements were validated using Spearman's rank correlation coefficient. The Spearman correlation is a nonparametric measure of rank correlation and assesses the strength of a monotonic relationship. Generally, Spearman's rank correlation describes the strength of correlation by using the following absolute values: $0.00-0.19$ as "very low"; $0.20-0.39$ as "low"; $0.40-0.59$ as "moderate"; 0.5-0.79 as "strong"; and $0.80-1.0$ as "very strong." The table below gives the comparison and the correlation that is calculated for that comparison depending on the variable type for the transverse processes of T3-T5. OMM, osteopathic manipulative medicine; US, ultrasound. for T4 SP. For comparison of ultrasonography vs. OMM overall, we found Fleiss' Kappa of -0.0623 for T2 SP, -0.0119 for T3 SP, and 0.0338 for T4 SP, indicating low levels of agreements (Table 1C). Spearman's rank correlation coefficients were all below 0.19 and consistent with the low levels of agreement calculated via Cohen's Kappa and Fleiss' Kappa (Table 1E).

\section{Discussion}

The purpose of this study is to evaluate a preliminary assessment of a protocol that examines the relationship between osteopathic palpation and ultrasonographic measurements of the thoracic spine. Our results show, at least for this particular protocol, that in an asymptomatic and generally healthy student population, a low level of interexaminer agreement between these two modalities for thoracic vertebrae levels T3-T5 and an overall lower range of agreement within each modality. Seeing that previous studies comparing OMM and ultrasonography utilized a single examiner within each modality, two examiners are utilized in our study to decrease single examiner bias and in the hopes of enhancing validity [24-26]. As expected, the osteopathic examination at T3-T5 indicated a low level of interexaminer reliability between OMM1 and OMM2, which is consistent with the literature when few examiners are utilized [12, 27, 35, 36]. In regard to US examination, the fair level of interexaminer agreement at prominent vertebral levels was reduced to a low level of interexaminer agreement when considering all vertebral levels. However, US examination, within the protocol of this study, did demonstrate slightly higher interexaminer agreement than osteopathic examination, as indicated by the Spearman's Rank correlation. This suggests that with additional adjustments to the protocol and further studies, a reliable modality including US is feasible.

It is worth noting that there was higher correlation within each modality and better agreement between both modalities at the T5 vertebral level. While this study was focused on asymptomatic participants, the higher correlation and agreement at vertebral level $\mathrm{T} 5$ is very promising in regard to utilizing a similar study design in the development of future studies. Pagé et al. [37] found that palpation and US of the spinous and transverse process of T5 had a higher validity and accuracy in vitro. This is consistent with the reported higher reliability for both osteopathic examiners and sonographers at T5. Considering the complexity of the thoracic spine, as well as the asymptomatic state of the participants in this study, it may 


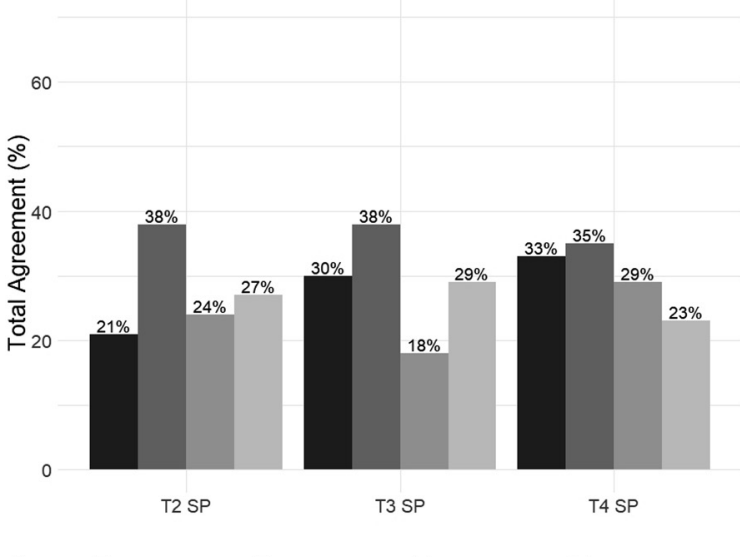

A
0.6

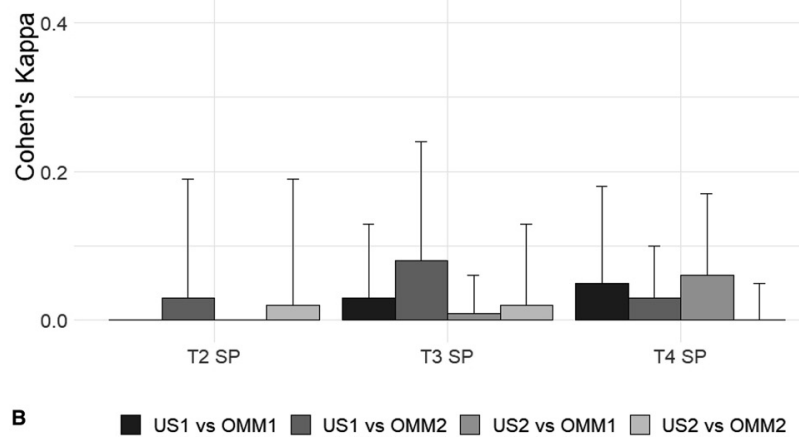

Figure 4: (A) Bar plot of the total agreement between OMM and US examiners, for each pairwise comparison of T2-T4 SPs. (B) Bar plot of Cohen's Kappa between OMM and US examiners, for each pairwise comparison at T2 SP, T3 SP, and T4 SP. The error bar is representative of the $95 \%$ confidence interval for Cohen's Kappa. We interpret Cohen's Kappa as $0.10-0.20$ is indicative of slight agreement, $0.21-0.40$ of fair agreement; $0.41-0.60$ of moderate agreement; $0.61-0.80$ of substantial agreement; and $0.81-0.99$ of near-perfect agreement. OMM, osteopathic manipulative medicine; SPs, spinous processes; US, ultrasound.

Table 1E. Spearman's rank correlation coefficients with SP as references for T2-T4 SP.

\begin{tabular}{|c|c|c|c|}
\hline \multirow{2}{*}{$\begin{array}{l}\text { Spinous } \\
\text { process }\end{array}$} & \multirow[t]{2}{*}{ Comparison } & \multicolumn{2}{|c|}{ Spearman correlation coefficient } \\
\hline & & $\begin{array}{l}\text { Correlation coefficient } \\
\text { ( } 95 \% \text { confidence interval) }\end{array}$ & p-Value \\
\hline \multirow[t]{4}{*}{$\mathrm{T} 2$} & $\begin{array}{l}\text { US1 vs. } \\
\text { OMM1 }\end{array}$ & NA & \\
\hline & $\begin{array}{l}\text { US1 vs. } \\
\text { OMM2 }\end{array}$ & $0.02(-0.22,0.26)$ & 0.8504 \\
\hline & $\begin{array}{l}\text { US2 vs. } \\
\text { OMM1 }\end{array}$ & NA & \\
\hline & $\begin{array}{l}\text { US2 vs. } \\
\text { OMM2 }\end{array}$ & $0.02(-0.22,0.26)$ & 0.8945 \\
\hline \multirow[t]{4}{*}{ T3 } & $\begin{array}{l}\text { US1 vs. } \\
\text { OMM1 }\end{array}$ & $0.03(-0.21,0.27)$ & 0.8105 \\
\hline & $\begin{array}{l}\text { US1 vs. } \\
\text { OMM2 }\end{array}$ & $0.08(-0.17,0.31)$ & 0.5321 \\
\hline & $\begin{array}{l}\text { US2 vs. } \\
\text { OMM1 }\end{array}$ & $-0.10(-0.34,0.14)$ & 0.4017 \\
\hline & $\begin{array}{l}\text { US2 vs. } \\
\text { OMM2 }\end{array}$ & $-0.04(-0.28,0.20)$ & 0.7297 \\
\hline \multirow[t]{4}{*}{$\mathrm{T} 4$} & $\begin{array}{l}\text { US1 vs. } \\
\text { OMM1 }\end{array}$ & $0.14(-0.11,0.37)$ & 0.2728 \\
\hline & $\begin{array}{l}\text { US1 vs. } \\
\text { OMM2 }\end{array}$ & $-0.02(-0.26,0.22)$ & 0.8562 \\
\hline & $\begin{array}{l}\text { US2 vs. } \\
\text { OMM1 }\end{array}$ & $0.18(-0.06,0.41)$ & 0.1401 \\
\hline & $\begin{array}{l}\text { US2 vs. } \\
\text { OMM2 }\end{array}$ & $-0.14(-0.37,0.11)$ & 0.2725 \\
\hline
\end{tabular}

Again, Spearman correlation assesses the strength of a monotonic relationship. Generally, Spearman's rank correlation describes the strength of correlation by using the following absolute values: $0.00-$ 0.19 as "very low", $0.20-0.39$ as "low", $0.40-0.59$ as "moderate", $0.5-0.79$ as "strong", and $0.80-1.0$ as "very strong". T2 cannot be calculated for some correlations because no rotations were noted at $\mathrm{T} 2$ by OMM1. OMM, osteopathic manipulative medicine; SP, spinous process; US, ultrasound. be worth examining the correlation between the two modalities before and after treatment of symptomatic somatic dysfunctions [17, 28, 32]. Further examination of anatomical changes after treatments will provide greater understanding on the pathophysiology of somatic dysfunctions and the mechanisms utilized to relieve them. However, this study was effective in providing groundwork and considerations when improving a study design that matches the modalities and clinical applications. Our results also suggest that application of this study design to a training protocol for osteopathic medical students might yield benefits at least in the lower vertebral segments where perhaps rotations and somatic dysfunctions are more common [24].

Regarding the vertebral levels that were found to have lower levels of agreement and correlation between US and osteopathic palpatory examination, we have identified a few possible explanations and limitations. First, as shown through teaching palpatory diagnosis in osteopathic medical students, one of the difficulties to palpation is that the students typically learn on other, generally healthy, asymptomatic students. This may lead to some of the difficulties seen in reliability of diagnosis and interexaminer reliability, even among advanced practitioners [12, 38-40]. In our study, for instance, the difference between the right and left TPs was measured to be an average of $2.07 \mathrm{~mm}$ in a healthy, asymptomatic, student population. Osteopathic physicians determined a left or right rotation based on their palpatory feedback, which may not always be prominent because the subjects were mostly asymptomatic. Efforts to standardize palpatory feedback were taken into consideration during protocol creation, and we utilized multiple training sessions prior to data collection to diminish the effects of palpatory feedback discrepancy. Given that 
Mancini et al. [41] showed statistically significant changes after OMT of $14.5 \mathrm{~mm}$ with US, one reason for the difference in interexaminer reliability seen in our study and some previous interexaminer studies may be that skilled osteopaths are often seeking to palpate larger differences than the ones measured in our study [10, 35, 41-44]. As such, the low interexaminer agreement within osteopathic palpatory examination may be best explained by the specifically chosen asymptomatic participants. However, the use of osteopathic palpatory examination in anatomical significant differences (i.e., somatic dysfunctions) is supported by the higher agreement at the T5 level. This possible connection emphasizes further exploration of osteopathic palpatory examination in the clinical setting-where somatic dysfunctions are common and requiring treatment.

Second, it is possible that the sample population and the size utilized in statistical analysis may not be representative of the efficacy of these two modalities and may have resulted in a diminished power that prevented a more accurate comparison of the modalities. However, the improved agreement at T5, even with a small sample size, is encouraging and does provide enough participants to create a study design that allows for a comparison between the two modalities in a scenario as standardized as possible while similarly overcoming single-examiner bias.

Finally, although every effort was made to limit rotation of the test population by asking them to look forward and limiting motion to flexion, it is certainly possible that the pressure of the palpating physicians' hands, as well as the pressure from the US probes, may have made slight alterations in the location of the TPs between the various examiners, making slight changes seen in our data decreasing interexaminer reliability. Furthermore, the palpation protocol artificially limited structural examination to palpation of the TPs and flexion, limiting the normal diagnostic ability to passively extend, rotate, and sidebend the patient to increase the confidence of the diagnosis $[45,46]$. US, on the other hand, is a static exam that requires the subject to remain motionless when diagnosing vertebral rotation $[1,47,48]$. As such, there may be an inherent and incompatible difference between US and osteopathic diagnostic examinations, which is supported by Snider et al. [26], where musculoskeletal changes in the sacral base identified by osteopathic palpatory examination could not be similarly identified by US examination. Considering the potential of this study design in a more clinically applicable sce nario (i.e., before and after treatment in asymptomatic and symptomatic patients), the relationships and considerations identified in this study could be instrumental in illumina ting important clinical relationships between osteopathic palpatory examination and ultrasonographic examination while further understanding the physiological mechanisms of somatic dysfunction and their appropriate treatments.

Another point to address is how the superior of two vertebra in a single segment may affect the findings of the vertebra below. A rotated vertebra changes the position of the TPs and SP such that the SP would be located more contralaterally to the posteriorly displaced TP and the direction of the vertebral rotation [34]. For instance, if T2 is rotated to the left, then the left $\mathrm{T} 2 \mathrm{TP}$ is more posterior and T2 SP is contralaterally displaced more to the right. This may lead to an inaccurate designation of rotation for the inferior vertebra, as the distance measured between the SP of the superior vertebra to the TP of the inferior vertebra is now altered. To address this phenomenon, we established an alternative analysis by utilizing the SP of each vertebra as our reference point instead of the TP of the inferior vertebra. To do this, we relabeled the ultrasonography measurement data so that it correlates with the SP of the superior vertebra (e.g., T4 TP data=T3 SP measurement). The OMM findings for SPs are also adjusted from the palpation findings of the TPs for statistical calculations (e.g., a palpated posterior T3 TP on the $\mathrm{L}=\mathrm{T} 3$ rotated to the $\mathrm{L}=\mathrm{T} 3 \mathrm{SP}$ displaced to the $\mathrm{R}$ ). Then, we compared the agreement for both ultrasonography and OMM findings of each SP of T2-T4. This alternative analysis does not have any effects on the interexaminer reliability within each modality (i.e., US1 vs. US2, OMM1 vs. OMM2) and resulted in no changes from our original findings. As such, further studies are needed to establish a true "neutral" on the patient and to compare all US findings to that static neutral point and may further enhance US as a training tool in the medical school setting.

To our knowledge, this is a novel study examining the thoracic spine utilizing US and structural surface palpation as utilized in osteopathic medicine. This study compares some observational techniques that are utilized clinically and utilizes US as a readily available modality to visualize anatomy in an in vivo, noninvasive, and safe manner. Although US demonstrated slightly higher agreement overall, generally, in a fast-paced clinical setting, it may not be realistic for physicians to evaluate the thoracic spine with US because of the extra time required with potentially low clinical yield. In the end, this study was designed to contribute to the growing literature surrounding osteopathic medicine with the hope that the methods and results reported may help contribute to the foundation of future studies examining osteopathic palpatory examination and treatments, as well as the role of ultrasonographic examination in clinical practice. 


\section{Conclusions}

In conclusion, this preliminary study of an asymptomatic population revealed, as shown via Cohen's Kappa and Fleiss' Kappa, that there is a low-to-moderate interexaminer reliability between sonographers, low-to-slight interexaminer reliability between osteopathic physicians, and low interexaminer reliability between OMM palpatory examination and ultrasonographic evaluation of the thoracic spine. There is, however, a slightly higher reliability at T5. The findings align with the main role of osteopathic palpatory examination as a clinical tool: the diagnosis of asymmetrical somatic dysfunction with the intention of treatment. This study was designed to contribute to the growing literature surrounding osteopathic medicine with the hope that the methods and results reported may help contribute to the foundation of future studies examining osteopathic palpatory examination and treatments, as well as the role of ultrasonographic examination in clinical practice and in teaching diagnostic palpation skills to osteopathic medical students. Future studies looking at US and OMM exam correlation on both asymptomatic and a clinically symptomatic population may prove to be an easier way to analyze validity and calibrate the two relationships between ultrasonography and OMM structural examination. Given that previous studies performed on other regions of the spine utilizing US to assess pre- and posttreatment measurements yielded positive and significant findings, further study of interexaminer reliability on the thoracic spine pre- and posttreatments may also yield significant findings that may prove beneficial to the osteopathic medical field.

Acknowledgments: The research team thanks Katherine A. Worden, DO, FAAO, and Gregory Heller, DO for their help in protocol creation. We thank Andy Huang, Montgomery Young, Molly Sturlis, and Varshini Venkatesan, for their help in data collection. This work was supported by Midwestern University and the Osteopathic Manipulative Medicine Department.

Research funding: None reported.

Author contributions: All authors provided substantial contributions to conception and design, acquisition of data, or analysis and interpretation of data. All authors drafted the article or revised it critically for important intellectual content. All authors gave final approval of the version of the article to be published; and all authors agree to be accountable for all aspects of the work in ensuring that questions related to the accuracy or integrity of any part of the work are appropriately investigated and resolved.
Competing interests: None reported.

Ethical approval: This study was registered with ClinicalTrials. gov (identifier: NCT04823637). This study was approved by the Institutional Review Board at Midwestern University in Glendale, Arizona (HHS IRB Registration: IRB00004085, Assurance Number: FWA00006635).

Informed consent: All participants provided written informed consent while completing their registration.

\section{References}

1. Heo JY, Lee JW, Kim CH, Lee SM, Choi YS. The validation of ultrasound-guided target segment identification in thoracic spine as confirmed by fluoroscopy. Clin Orthop Surg 2017;9:472.

2. Leboeuf-Yde C, Nielsen J, Kyvik KO, Fejer R, Hartvigsen J. Pain in the lumbar, thoracic or cervical regions: do age and gender matter? A population-based study of 34,902 Danish twins 20-71 years of age. BMC Musculoskelet Disord 2009;10:39.

3. Lenehan KL, Fryer G, McLaughlin P. The effect of muscle energy technique on gross trunk range of motion. J Osteopath Med 2003; 6:13-8.

4. Licciardone JC, Nelson KE, Glonek T, Sleszynski SL, Cruser DA. Osteopathic manipulative treatment of somatic dysfunction among patients in the family practice clinic setting: a retrospective analysis. J Am Osteopath Assoc 2005;105:537-44.

5. Minarini G, Ford M, Esteves J. Immediate effect of T2, T5, T11 thoracic spine manipulation of asymptomatic patient on autonomic nervous system response: single-blind, parallel-arm controlled-group experiment. Int J Osteopath Med 2018;30: 12-7.

6. Campbell SM, Winkelmann RR, Walkowski S. Osteopathic manipulative treatment: novel application to dermatological disease. J Clin Aesthetic Dermatol 2012;5:24-32.

7. DiGiovanna EL, Amen CJ, Burns DK. An osteopathic approach to diagnosis and treatment, 4th ed. Philadelphia, PA: Wolters Kluwer; 2020.

8. Seffinger MA. American Osteopathic Association, editors. Foundations of osteopathic medicine: philosophy, science, clinical applications, and research, 4th ed. Philadelphia: Wolters Kluwer; 2018.

9. Brindise J, Nelson K, Kappler R. Association between cervical and thoracic somatic dysfunction among second-year osteopathic medical students. J Am Osteopath Assoc 2014;114:540-8.

10. Degenhardt BF, Johnson JC, Snider KT, Snider EJ. Maintenance and improvement of interobserver reliability of osteopathic palpatory tests over a 4-month period. J Am Osteopath Assoc 2010;110:579-86.

11. Snider EJ, Pamperin K, Pazdernik V, Degenhardt BF. Influence of transverse process landmark localization on palpation accuracy of lumbar spine models. J Am Osteopath Assoc 2018;118:151.

12. Bengaard K, Bogue RJ, Crow WT. Reliability of diagnosis of somatic dysfunction among osteopathic physicians and medical students. Osteopath Fam Physician 2012;4:2-7.

13. Oakley CK, Janssen SAK, Pankratz JP, McCumber TL, Treffer KD, Olinger AB. Validity of the rule of threes and anatomical relationships in the thoracic spine. J Am Osteopath Assoc 2018; 118:645. 
14. Mitchell F, Moran P, Pruzzo N. An evaluation and treatment manual of osteopathic muscle energy procedures, 1st ed. Valley Park, MO: Mitchell, Moran, and Pruzzo Associates; 1979.

15. Geelhoed MA, McGaugh J, Brewer PA, Murphy D. A new model to facilitate palpation of the level of the transverse processes of the thoracic spine. J Orthop Sports Phys Ther 2006;36:876-81.

16. Chin KJ, Karmakar MK, Peng P. Ultrasonography of the adult thoracic and lumbar spine for central neuraxial blockade. Anesthesiology 2011;114:1459-85.

17. Willems J, Jull G, Ng J-F. An in vivo study of the primary and coupled rotations of the thoracic spine. Clin Biomech 1996;11: $311-6$.

18. Lockwood MD, Kondrashova T, Johnson JC. Feasibility of using ultrasonography to establish relationships among sacral base position, sacral sulcus depth, body mass index, and sex. J Am Osteopath Assoc 2015;115:648.

19. Czyrny Z. Standards for musculoskeletal ultrasound. J Ultrason. 2017;17:182-7.

20. Ahmed AS, Ramakrishnan R, Ramachandran V, Ramachandran SS, Phan K, Antonsen EL. Ultrasound diagnosis and therapeutic intervention in the spine. J Spine Surg 2018;4:423-32.

21. Ledsome JR, Lessoway V, Susak LE, Gagnon FA, Gagnon R, Wing $P C$. Diurnal changes in lumbar intervertebral distance, measured using ultrasound. Spine 1996;21:1671-5.

22. Patil P, Dasgupta B. Role of diagnostic ultrasound in the assessment of musculoskeletal diseases. Ther Adv Musculoskelet Dis 2012;4:341-55.

23. Flaum TB, Rusnack FM, Mirza A, Apoznanski TE, Munarova A, Mazzie JP, et al. An observational study of ultrasound to confirm cervical spine segmental positional rotation. Int J Osteopath Med 2017;25:1-5.

24. Winter J, Kimber A, Montenegro S, Gao J. Ultrasonography to assess the efficacy of osteopathic manipulative treatment for lumbar spine asymmetry. J Am Osteopath Assoc 2020;120:761.

25. Shaw KA, Dougherty JJ, Treffer KD, Glaros AG. Establishing the content validity of palpatory examination for the assessment of the lumbar spine using ultrasonography: a pilot study. J Am Osteopath Assoc 2012;112:775.

26. Snider KT, Redman CL, Edwards CR, Bhatia S, Kondrashova T. Ultrasonographic evaluation of the effect of osteopathic manipulative treatment on sacral base asymmetry. J Am Osteopath Assoc 2018;118:159.

27. Beynon AM, Hebert JJ, Walker BF. The interrater reliability of static palpation of the thoracic spine for eliciting tenderness and stiffness to test for a manipulable lesion. Chiropr Man Ther 2018;26:49.

28. Morita D, Yukawa Y, Nakashima H, Ito K, Yoshida G, Machino M, et al. Range of motion of thoracic spine in sagittal plane. Eur Spine J 2014;23:673-8.

29. Savarese RG, Capobianco JD, Adesina AT, Reed G. OMT Review: a comprehensive review in osteopathic medicine, 4th ed. Florida, USA: OMT Review LLC; 2018.

30. Moore KL, Agur AMR, Dalley AF. Clinically oriented anatomy, 8th ed. Philadelphia: Wolters Kluwer; 2018.

31. Stroud L, Herold J, Tomlinson G, Cavalcanti RB. Who you know or what you know? Effect of examiner familiarity with residents on OSCE scores. Acad Med 2011;86:S8-11.

32. Csernátony Z, Molnár S, Hunya Z, Manó S, Kiss L. Biomechanical examination of the thoracic spine-the axial rotation moment and vertical loading capacity of the transverse process: transverse process loading capacity. J Orthop Res 2011;29:1904-9.

33. Akoglu H. User's guide to correlation coefficients. Turk J Emerg Med 2018;18:91-3.

34. Lam GC, Hill DL, Le LH, Raso JV, Lou EH. Vertebral rotation measurement: a summary and comparison of common radiographic and CT methods. Scoliosis 2008;3:16.

35. Consorti G, Basile F, Pugliese L, Petracca M. Interrater reliability of osteopathic sacral palpatory diagnostic tests among osteopathy students. J Am Osteopath Assoc 2018;118:637.

36. Walker BF, Koppenhaver SL, Stomski NJ, Hebert JJ. Interrater reliability of motion palpation in the thoracic spine. Evid Based Complement Alternat Med 2015;2015:1-6.

37. Pagé I, Descarreaux M, Sobczak S. Development of a new palpation method using alternative landmarks for the determination of thoracic transverse processes: an in vitro study. Musculoskelet Sci Pract 2017;27:142-9.

38. Wilke H-J, Herkommer A, Werner K, Liebsch C. In vitro analysis of the segmental flexibility of the thoracic spine. PLoS one 2017;12: e0177823.

39. Stochkendahl MJ, Christensen HW, Hartvigsen J, Vach W, Haas M, Hestbaek L, et al. Manual examination of the spine: a systematic critical literature review of reproducibility. J Manip Physiol Ther 2006;29:475-85. e10.

40. Vaughan B. Inter-examiner reliability in detecting cervical spine dysfunction: a short review. J Osteopath Med 2002; 5:24-7.

41. Mancini D, Cesari M, Lunghi C, Benigni AM, Antonelli Incalzi R, Scarlata S. Ultrasound evaluation of diaphragmatic mobility and contractility after osteopathic manipulative techniques in healthy volunteers: a prospective, randomized, double-blinded clinical trial. J Manip Physiol Ther 2019;42:47-54.

42. Haneline MT, Young M. A review of intraexaminer and interexaminer reliability of static spinal palpation: a literature synthesis. J Manip Physiol Ther 2009;32:379-86.

43. Potter L, McCarthy C, Oldham J. Intraexaminer reliability of identifying a dysfunctional segment in the thoracic and lumbar spine. J Manip Physiol Ther 2006;29:203-7.

44. Lucas N, Bogduk N. Diagnostic reliability in osteopathic medicine. Int J Osteopath Med 2011;14:43-7.

45. Walkowski S, Baker R. Osteopathic manipulative medicine. In: pain procedures in clinical practice. Philadelphia: Elsevier; 2011: 155-71 pp.

46. Gibbons P, Tehan P. Muscle energy concepts and coupled motion of the spine. Man Ther 1998;3:95-101.

47. Porto AB, Okazaki VHA. Thoracic kyphosis and lumbar lordosis assessment by radiography and photogrammetry: a review of normative values and reliability. J Manip Physiol Ther 2018;41: 712-23.

48. Øverås CK, Myhrvold BL, Røsok G, Magnesen E. Musculoskeletal diagnostic ultrasound imaging for thickness measurement of four principal muscles of the cervical spine -a reliability and agreement study. Chiropr Man Ther 2017;25:2.

Supplementary Material: The online version of this article offers supplementary material (https://doi.org/10.1515/jom-2021-0020). 